\title{
Clinical Value of Musculoskeletal Ultrasound in Rehabilitation After Flexor Tendon Rupture Repair of the Hand
}

\author{
Rui Wang* \\ Yancheng Third People's Hospital, Yancheng 224000, Jiangsu Province, China \\ *Corresponding author: Rui Wang, 66981001@qq.com
}

Copyright: (C) 2022 Author(s). This is an open-access article distributed under the terms of the Creative Commons Attribution License (CC BY 4.0), permitting distribution and reproduction in any medium, provided the original work is cited.

\begin{abstract}
Objective: To explore the application effect and clinical value of musculoskeletal ultrasound in the rehabilitation of hand function after flexor tendon rupture repair. Methods: In this study, 72 patients were selected from among patients who underwent flexor tendon rupture repair of the hand in Yancheng Third People's Hospital from May 2018 to May 2020; the patients were randomly divided into the control group (routine hand rehabilitation training) and the experimental group (musculoskeletal ultrasound and targeted hand rehabilitation training based on examination results) by die roll, with 34 cases in each group; the hand rehabilitation of the two groups were compared. Results: The excellent and good rate of the total active motion (TAM) of the experimental group (94.44\%) was significantly higher than that of the control group (69.44\%) $(P$ $<0.05$ ); before treatment, there was no significant difference in the diameter and degree of stenosis of the artery in the finger between the two groups $(P>0.05)$; after treatment, the degree of stenosis and the diameter of the artery of the experimental group were significantly better than those of the control group $(P<0.05)$. Conclusion: For patients treated with flexor tendon rupture repair of the hand, the use of musculoskeletal ultrasound in the rehabilitation process can significantly improve the functional recovery of the hand; therefore, it is worthy of in-depth research, promotion, and application in clinical rehabilitation.
\end{abstract}

Keywords: Musculoskeletal ultrasound; Hand; Flexor tendon rupture; Repair; Hand rehabilitation; Clinical value

Online publication: January 19, 2022

\section{Introduction}

The rupture of flexor tendon in the hand is mainly caused by strong external forces. Timely and effective treatment is required upon rupture. The general treatment for this condition is by repair. Rehabilitation training is needed after surgery; otherwise, the patient may have serious dysfunction to his or her daily life in the future. In postoperative rehabilitation training, patients need to aim for good tendon healing. In routine rehabilitation training, rehabilitation personnel only train based on prior clinical experience, without any targeted rehabilitation in consideration of the patients' actual situation; therefore, the effect has not significantly improved ${ }^{[1]}$. With the continuous development of science and technology, the diagnostic process has improved. Among them, diagnostic ultrasound can effectively diagnose tendon rupture by using high-frequency ultrasound as well as evaluate the state and healing of tendons. Therefore, 72 patients, who underwent flexor tendon rupture repair of the hand, were selected from Yancheng Third People's Hospital to explore the application effect of musculoskeletal ultrasound in postoperative rehabilitation. 


\section{Data and methods}

\subsection{General information}

Seventy-two patients with flexor tendon rupture of the hand treated in Yancheng Third People's Hospital from May 2018 to May 2020 were selected as the subjects in this study. The patients were divided by die roll into two groups, the control group and the experimental group, with 34 cases in each group. In the control group, there were 21 male patients and 13 female patients, age ranging from 22 to 53 years old, with an average age of $33.25 \pm 8.76$ years old. In the experimental group, there were 23 male patients and 11 female patients, age ranging from 20 to 51 years old, with an average age of $33.65 \pm 7.89$ years old. There was no significant difference in age, sex, and other general data between the two groups $(P>0.05)$.

The inclusion criteria were as follows: (1) patients with flexor tendon rupture of the hand; (2) those without obvious symptoms of hand infection and combined injury; (3) those who signed the informed consent form of this study; (4) those who received rehabilitation within one month after the repair ${ }^{[2]}$.

The exclusion criteria were as follows: (1) patients with combined injury of other parts; (2) those with severe mental dysfunction or mental illness; (3) those with low compliance and unable to cooperate with rehabilitation training.

\subsection{Method}

The patients in the control group received routine rehabilitation training of the hand, and the results of the hand function evaluation scale were used as the basis of rehabilitation training. Three days after the repair, the external fixation plaster was removed in consideration of the actual situation of the patients, and the patients were guided to carry out mild active flexion and extension training. One month after the repair, they were trained in non-resistance active flexion and extension. Anti-resistance active flexion and extension exercise was performed 6 weeks after the repair ${ }^{[3]}$.

For the experimental group, musculoskeletal ultrasound was used before the initiation of rehabilitation training. Upon obtaining the examination results, a scientific, reasonable, and targeted hand rehabilitation training plan was constructed based on the results. At 2 weeks, 4 weeks, 6 weeks, 8 weeks, 12 weeks, and 16 weeks after the repair, the patients were regularly examined by musculoskeletal ultrasound. According to the inspection of the structures each time, the training is adjusted accordingly. The instrument used was a digital color doppler ultrasonic diagnostic instrument, and the probe frequency was set to 4-13 MHz. For each patient, the inspection process was as follows: (1) the tendon was continuously scanned with the probe; (2) the tendon was scanned longitudinally. During scanning, the tissue size, marginal tissue, specific structure, and the internal echo of the lesion were determined. The specific ultrasonographic changes to the injured flexor tendon suture site and active scar connection were observed. In case of tendon adhesion, it is necessary to determine the moving parts and formulate a targeted hand rehabilitation training plan based on the inspection results ${ }^{[4]}$.

\subsection{Observation indicators}

(1) Overall efficacy of total active motion (TAM)

It is divided into four grades: excellent, good, medium, and poor. If the range of motion is normal, it is deemed as excellent; compared with the healthy side, TAM $\geq 75 \%$ is considered good, $50 \% \leq$ $\mathrm{TAM}<75 \%$ is considered as medium, and TAM $<50 \%$ is deemed as poor ${ }^{[5]}$.

(2) Inner diameter of artery and degree of artery stenosis 


\subsection{Statistical analysis}

The data in this study were processed and calculated by SPSS 24.0. The counting data were expressed in $\mathrm{n} / \%$ and tested by $X^{2}$; the measurement data were expressed in $\overline{\mathrm{x}} \pm \mathrm{s}$ and tested by $t . P<0.05$ indicates a statistically significant difference.

\section{Results}

\subsection{TAM effect}

The TAM effect of the experimental group was significantly better than that of the control group $(\mathrm{P}<0.05)$, as shown in Table 1.

Table 1. Comparison of the TAM effect between the two groups [n (\%)]

\begin{tabular}{cccccc}
\hline Group & Excellent & Good & Medium & Poor & Excellent and good rate \\
\hline Experimental group $(\mathrm{n}=36)$ & $20(55.56)$ & $14(38.89)$ & $2(5.56)$ & $0(0.00)$ & $34(94.44)$ \\
Control group $(\mathrm{n}=36)$ & $13(36.11)$ & $12(33.33)$ & $6(16.17)$ & $5(13.89)$ & $25(69.44)$ \\
$X^{2}$ value & & & & 7.6037 \\
$P$ value & & & & 0.0058 \\
\hline
\end{tabular}

\subsection{Diameter of the artery in the finger}

Before treatment, there was no significant difference in the diameter of the artery between the two groups $(P>0.05)$. After treatment, the arterial diameter of the two groups significantly improved, but the arterial diameter of the experimental group was significantly better than that in the control group $(P<0.05)$, as shown in Table 2.

Table 2. Comparison of arterial diameter of the finger between the two groups before and after treatment $(\overline{\mathrm{x}} \pm \mathrm{s})$

\begin{tabular}{ccc}
\hline Group & \multicolumn{2}{c}{ Diameter of artery $(\mathrm{mm})$} \\
\cline { 2 - 3 } & Before treatment & After treatment \\
\hline Experimental group $(\mathrm{n}=36)$ & $0.87 \pm 0.01$ & $1.18 \pm 0.04$ \\
Control group $(\mathrm{n}=36)$ & $0.87 \pm 0.02$ & $1.01 \pm 0.06$ \\
$X^{2}$ value & 0.0000 & 14.1449 \\
$P$ value & 1.0000 & 0.0000 \\
\hline
\end{tabular}

\subsection{Degree of stenosis of the artery in the finger}

Before treatment, there was no significant difference in the degree of stenosis between the two groups $(P>$ 0.05). After treatment, the degree of stenosis of the two groups significantly improved, but the degree of stenosis of the experimental group was significantly better than that of the control group $(P<0.05)$, as shown in Table 3. 
Table 3. Comparison of the degree of stenosis of the artery in the finger between the two groups before and after treatment $(\overline{\mathrm{x}} \pm \mathrm{s})$

\begin{tabular}{ccc}
\hline Group & \multicolumn{2}{c}{ Degree of stenosis (\%) } \\
\cline { 2 - 3 } & Before treatment & After treatment \\
\hline Experimental group $(\mathrm{n}=36)$ & $69.98 \pm 9.06$ & $42.39 \pm 5.77$ \\
Control group $(\mathrm{n}=36)$ & $67.39 \pm 9.23$ & $52.74 \pm 6.82$ \\
$X^{2}$ value & 1.2015 & 6.9515 \\
$P$ value & 0.2336 & 0.0000 \\
\hline
\end{tabular}

\section{Discussion}

The flexor tendon of the hand is a very important tissue structure in the human hand. As tendons are important for joint activity, they play important roles in people's daily life and activity. If the flexor tendon of the hand is damaged, it will lead to the loss of normal activity and function of the hand. At present, with the continuous development of urbanization, the probability of injury is gradually increasing due to various factors, such as high-intensity work. Combined with relevant statistical data, tendon injury accounts for more than $30 \%$ of hand injuries, and patients receiving flexor tendon repair account for more than $90 \%$ of patients with tendon injuries ${ }^{[6]}$. Flexor tendon rupture of the hand is a trauma caused by external forces, which is commonly seen in clinical practice. Upon rupture, the finger is presented in a flexion grip form. If the finger joint is straightened, the distal tendon will move with it. As a result, it is difficult to repair the broken end in the actual process of flexor tendon repair, and even if the repair is successful, the tendon may rupture again after suturing. Therefore, it is necessary to understand the state of the ruptured tendon in advance. At present, diagnostic ultrasound is widely used in clinics and is gradually maturing in China. Musculoskeletal ultrasound is one of the diagnostic ultrasound techniques, which has the characteristics of high probe frequency and clear image display [7]. In postoperative rehabilitation, patients can fully understand the tendon connection in the hand and determine the specific state of tendon healing through musculoskeletal ultrasound before formulating a rehabilitation training plan.

For patients after flexor tendon rupture repair of the hand, it is necessary to first carry out musculoskeletal ultrasound examination, and then formulate a postoperative rehabilitation training plan in consideration of the examination results. Compared with routine postoperative rehabilitation training, it can significantly improve the total active motion. For example, the results of this study showed that the excellent and good rate of TAM of the experimental group was $94.44 \%$, while that of the experimental group was $69.44 \%$, with statistically significant difference $(P<0.05)$. This shows that the application value of musculoskeletal ultrasonography is justified. The diameter and degree of stenosis of the artery in the finger of the experimental group were significantly better than those of the control group $(P<0.05)$. It also shows the recovery of the tendon after repair and before rehabilitation training. By carrying out musculoskeletal ultrasound examination first, and then formulating a rehabilitation training plan, there would be improvement in the quality of rehabilitation training and the recovery level of the tendon after repair.

In conclusion, for patients with flexor tendon rupture of the hand, formulating a targeted rehabilitation training plan based on the musculoskeletal ultrasound examination results after surgery can improve the speed of functional recovery. It has certain application value in clinical rehabilitation.

\section{Disclosure statement}

The author declares that there is no conflict of interest. 


\section{References}

[1] Qiu H, 2019, Clinical Nursing of Early Functional Rehabilitation after Repair of Flexor Tendon Rupture in Zone II of Hand. Chongqing Medicine, 48(A01): 2.

[2] Wan F, Cai Q, Lv L, 2019, Effect of Sodium Hyaluronate after Repair of Flexor Tendon Rupture of Hand. Medical Journal of National Defending Forces in Southwest China, 29(1): 3.

[3] Li C, Zhang D, Qian X, 2021, Application Value of High Frequency Ultrasound after Repair of Finger Tendon Rupture. Chinese Journal of Medical Ultrasound, 18(01): 88-91.

[4] Huang W, Xiao T, Li Y, et al., 2020, Effect of Electroacupuncture on Hand Functional Rehabilitation after Repair of Flexor Tendon Rupture. Reflexology and Rehabilitation Medicine, 2020(19): 3.

[5] Zhou F, Wang L, Xie G, et al., 2019, Application of Muscle Bone Ultrasound in Hand Functional Rehabilitation After Repair of Flexor Tendon Rupture. Journal of Imaging Research and Medical Application, 2019(15): 3.

[6] Huang W, Xiao T, Li Y, et al., 2020, The Clinical Value of Musculoskeletal Ultrasound in Hand Functional Rehabilitation after Flexor Tendon Fracture Repair. Reflexology and Rehabilitation Medicine, 2020(10): 2.

[7] Fan Y, Du H, 2020, Effect of Early Functional Exercise after Emergency Repair of Flexor Tendon Rupture of Hand. Yishou Baodian, 2020(18): 1. 